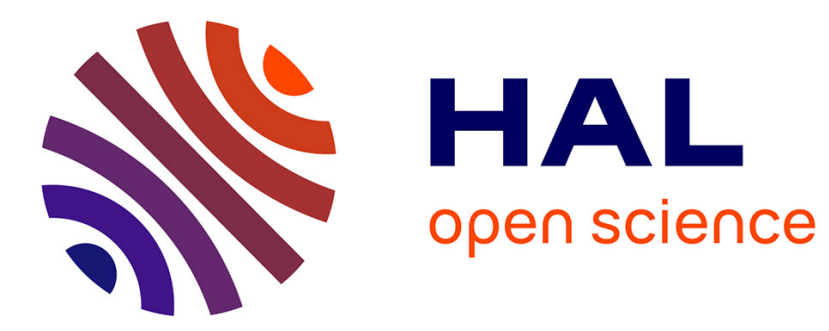

\title{
Development of a Generic Voter under FoCal
}

Philippe Ayrault, Thérèse Hardin, François Pessaux

\section{To cite this version:}

Philippe Ayrault, Thérèse Hardin, François Pessaux. Development of a Generic Voter under FoCal. TAP 2009 - 3rd International Conference on Tests and Proofs, Jul 2009, Zurich, Switzerland. pp.10-26, 10.1007/978-3-642-02949-3_3 . hal-01241361

\section{HAL Id: hal-01241361 https://hal.science/hal-01241361}

Submitted on 11 Dec 2015

HAL is a multi-disciplinary open access archive for the deposit and dissemination of scientific research documents, whether they are published or not. The documents may come from teaching and research institutions in France or abroad, or from public or private research centers.
L'archive ouverte pluridisciplinaire HAL, est destinée au dépôt et à la diffusion de documents scientifiques de niveau recherche, publiés ou non, émanant des établissements d'enseignement et de recherche français ou étrangers, des laboratoires publics ou privés. 


\title{
Development of a Generic Voter under FoCaL*
}

\author{
Philippe Ayrault ${ }^{1}$, Thérèse Hardin ${ }^{2}$, and François Pessaux ${ }^{2}$ \\ ${ }^{1}$ Etersafe, 43, Allée du pont des beaunes F-91120 Palaiseau \\ philippe.ayrault@etersafe.com \\ 2 Semantics, Proofs and Implementation, Laboratoire Informatique de Paris 6 \\ Pierre \& Marie Curie University, F-75005 Paris \\ therese.hardin|francois.pessaux@lip6.fr
}

\begin{abstract}
Safety and security are claimed major concerns by the formal FoCaL development environment. In [7] we introduced a safety development cycle customised to FoCaL. In this paper, we examine how to specify and implement a concrete example following this cycle. We show that indeed it is feasible and we present how FoCaL features fit with software best practises like modularity, reuse, fault confinement and maintenance.
\end{abstract}

Key words: formal methods, development cycle, voter, FoCaL

\section{Introduction}

Development of safety related systems has to be strictly compliant with applicable standards. This is required by any safety authority before commissioning of a system. Safety demonstration is greatly helped by using a formally based framework to express requirements, design, code and to ensure that this code meets specification requirements. Unfortunately, this is not sufficient. Safety authorities also require an independent verification process which must follow the different stages of a strict development cycle. Thus, any tool claiming to be dedicated to formal developments should not only provide a formal paradigm to model and prove the system but must also strongly support a well defined development cycle and must produce adequate documentation at each stage of the development. Moreover, such a tool should help to identify impacts of modifications during the whole life of the system (frequently more than 20 years). The purpose of the FoCaL tool is to bring some answers to this problem area. FoCaL provides a unified language to express requirements (declaration and properties) as well as source code and proofs while only using concepts largely accessible to engineers.

In [7], we introduced a safety development cycle customised for FoCaL. Our problem now is to study its feasibility and to understand how practically FoCaL can help to answer safety and good practise requirements. We have chosen to fully treat the example of a voter. Indeed a voter is a central equipment of all redundant architectures, widely used for safety related systems. A voter is a process or a device whereby a number of similar signals are monitored for discrepancies and are voted upon to obtain the

\footnotetext{
* This work is supported in part by the Agence Nationale de la Recherche under grant ANR-06SETI-016 for the SSURF Project (Safety and Security UndeR FoCaL).
} 
selected output which is most probably correct. It is highly safety critical because in many cases, the voter is the last barrier able to eliminate failure effects and answers a part of the safety principles underlining the whole system. As these ones strongly depend of the aim and domain of the system, for the re-usability sake, a voter should be conceived as a very generic equipment. Thus choosing a voter seems a good balance between the exemplary nature of the development and the length of the paper.

The rest of the paper is organised as follows. We present the main features of the FoCaL tool in Section 2. Section 3 exposes the rules of a voter and its textual specification. The Section 4 comments the development of the voter using the FoCaL tool. We conclude and comment possible further works in Section 5.

\section{The FoCaL environment}

We give here an informal presentation of near all FoCaL features, to help further reading of this paper. For more details and the new release ${ }^{3}$.

\subsection{The Basic Brick}

The primitive entity of a FoCaL development is the species. Like in most modular systems (i.e. objected oriented, algebraic abstract types), it can be viewed as a record grouping a data structure with its related operations. Since FoCaL does not only address data type and operations, species may contain the declarations (specifications) of these operations, some properties (which may represent requirements) and their proofs. All these components of a species are called methods and we briefly describe them.

- The method introduced by the keyword representation gives the data representation of entities manipulated by the species. It is defined by a type expression, which is roughly a ML-like pure type (with restricted polymorphism). The representation may be not-yet-defined in a species, meaning that the real structure of the data-type the species embeds does not need to be known at this point. However, to obtain an implementation, the representation has to be defined later either explicitly or by inheritance.

- Declarations (keyword signature followed by a name and a type) introduce methods to be defined later: they only specify types without implementation yet. Declarations serve to express specifications, properties. Thanks to late-binding, as soon as a name is declared, it can be used in definitions.

- Definitions (keyword let, followed by a name, a type and an expression) introduce constants or functions, i.e. computational operations. The expressions are roughly pure ML-like expressions with an auxiliary construction (S ! m) to call the method $\mathrm{m}$ from a given species $\mathrm{S}$.

- Statements (keyword property followed by a name and a first-order formula) may serve to express requirements (i.e. facts that the system must hold to conform to the Statement of Work) and then can be viewed as a specification purpose method, like signatures were for let-methods. They entail a proof obligation later in the development. Like signatures, even if no proof is yet given, the name of the property can be used to prove other theorems or lemmas.

${ }^{3}$ see : http://focalize.inria.fr 
- Theorems (theorem) made of a name, a statement and a proof are properties together with the formal proof that their statement holds in the context of the species. This proof will be processed by FoCaL and ultimately checked with the theorem prover Coq.

In addition, FoCaL provides a powerful mechanism for documentation by allowing special kind of commentaries (called annotations) kept along the compiler process.

\subsection{Type of Species, Interfaces and Collections}

The type of a species is obtained by removing definitions and proofs. Thus, it is a kind of record type, made of all the method types of the species. If the representation is still a type variable say $\alpha$, then the species type is prefixed with an existential binder $\exists \alpha$. This binder will be eliminated as soon as the representation will be instantiated (defined) and must be eliminated to obtain executable code. Species types remain totally implicit to users and serve only to introduce interfaces.

The interface of a species is obtained by abstracting the representation type in the species type and this abstraction, which hides the representation, is permanent. Interfaces play an important role. They are simply denoted by the species name. Interfaces can be ordered by inclusion, a point providing a very simple notion of sub-typing.

A species is said to be complete if all declarations have received definitions and all properties have received proofs. When complete, a species can be submitted to an abstraction process of its representation to create a collection. Thus the interface of the collection is just the interface of the complete species underlying it. A collection can hence be seen as an abstract data-type, only usable through the methods of its interface, but with the guarantee that all methods/theorems are defined/proved.

\subsection{Combining Bricks}

A FoCaL development is organised as a hierarchy which may have several roots. Usually the upper levels of the hierarchy are built during the specification stage while the lower ones correspond to implementation. Each node of the hierarchy, i.e. each species (or collection as terminal ends), is a progress to a complete implementation. There are two ways to build new species from previously built species: inheritance and parametrisation.

In FoCaL inheritance serves two kinds of evolutions, which can be freely mixed. One may create a new species by extending the inherited ones with new operations and properties while keeping those of the inherited ones (or redefining some of them). One may create a new species by giving explicit definitions to signatures and proofs to properties of the inherited species, to be closer to a "executable" implementation.

Multiple inheritance is available in FoCaL. In case of inheriting a method from several parents, the order of parents in the inherits clause serves to determine the chosen method.

The type of a species built using inheritance is defined like for other species, the methods types retained inside it being those of the methods present in the species after inheritance is resolved. 
A strong constraint in inheritance is that the type of inherited, and/or redefined methods must not change. This is required to ensure logical consistency of the FoCaL model.

FoCaL allows two flavors of parametrisation: parametrisation by collection parameters and parametrisation by entity parameters. For instance a pair is a structure which is built upon its two components and is described by a species parametrised by its two components. Entity parameters are not introduced as we do not use them within this paper.

The parametrised species can use collection parameters' methods to define its own ones. A collection parameter is given a name $C$ and an interface $I$. The name $C$ serves to call the methods of $C$ which figure in $I$. $C$ can be instantiated by an effective parameter $C E$ of interface $I E$. $C E$ is a collection and its interface $I E$ must contain $I$. Note that species (without abstraction) are not allowed as parameters. Indeed, if an incomplete species were used as an effective parameter run-time error due to linkage of libraries can occur and properties stated in $I$ can not be safely used as an hypothesis. In contrast, the collection and late-binding mechanisms ensure that all methods appearing in $I$ are indeed implemented in $C E$.

\subsection{The Final Brick}

As briefly introduced, a species needs to be complete to lead to executable code for its functions and checkable proofs for its theorems and then, can be turned into a collection. Hence, a collection represents the final stage of the inheritance tree of a species and leads to an effective data representation with executable functions processing it. As said before, to ensure modularity and abstraction, the representation of a collection turns hidden. This means that any software component dealing with a collection will only be able to manipulate it through the operations (methods) its interface provides. This point is especially important since it prevents other software components from possibly breaking invariants required by the internals of the collection.

\subsection{Properties, Theorems and Proofs}

FoCaL intends to encompass both the executable model (i.e. program) and properties this model must satisfy. For this reason, theorems, properties and proofs are methods dealing with logical instead of purely behavioural aspects of the system. Stating a property entails that a proof of it will be finally built. For theorems, the proof is directly embedded in the theorem. The compilation process submits proofs to the formal proof assistant Coq which automatically checks that they are consistent.

No syntax is offered to express high-order properties as they are rather difficult to manage by engineers not experts in logic theory. But special instances for induction and termination proofs are to be provided.

Proofs are done by the developer as follows. It can be written in "FoCaLProof Language", a hierarchical proof language that allows to give hints and directions for a proof. This script is submitted to an external theorem prover, Zenon ${ }^{4}$ developed by D. Doligez. Zenon is a first order theorem prover based on the Tableaux method incorporating implementation novelties such as sharing[4]. From these hints Zenon attempts

${ }^{4}$ see : http://focal.inria.fr/zenon/ 
to automatically generate a proof and if it succeeds, expresses its proof as a Coq term verified by Coq during the compilation process. Basic hints given by the developer to Zenon are: "prove by definition of a method" (i.e. looking inside its body) and "prove by property" (i.e. using the logical statement of a theorem or property". Surrounding this hints mechanism, the language allows to build the proof by stating assumptions (that must obviously be demonstrated next) which can be used to prove lemmas or parts for the whole property. We show below an example of such a demonstration.

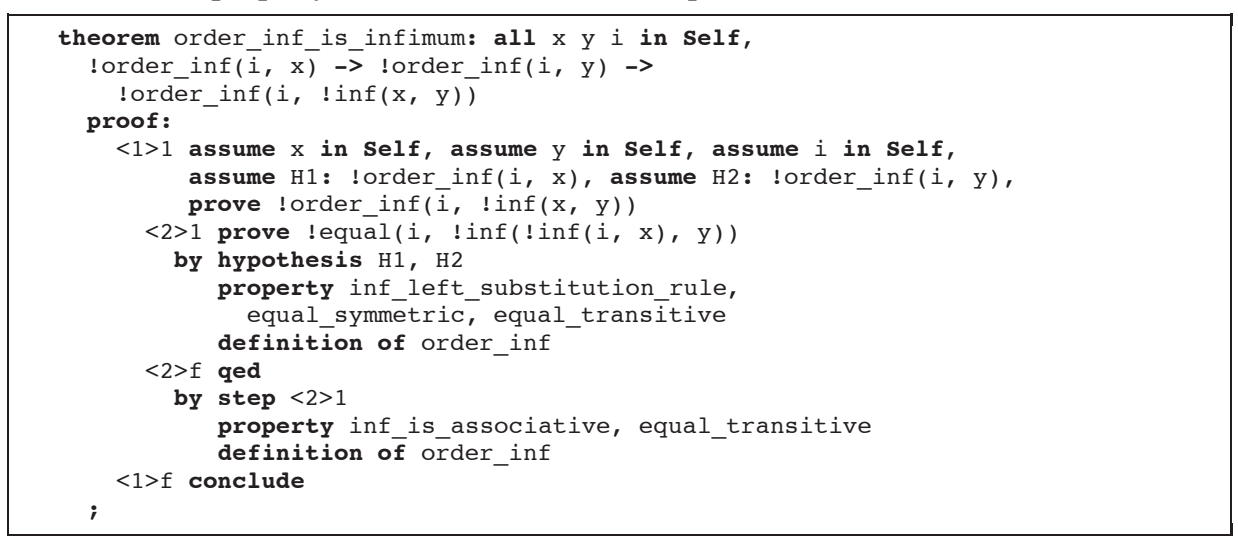

Like any automatic theorem prover, Zenon may fail finding a demonstration. In this case, FoCaL allows to write verbatim Coq proofs. The compiler provides a Coq script "with a hole" which can be filled with the proof done by hand, then imported back to the FoCaL source code as verbatim Coq code.

Finally, the assumed keyword is the ultimate proof backdoor, telling that no proof is given thus the property is considered as an axiom. Obviously, a really safe development should not make usage of such "proofs" since they bypass the formal verification of software's model and can break the global consistency. However, a development may use external (trusted or not) code no property of which can be proved. Moreover, whatever the reason, the user may choose to admit some properties. But any "assumed" lemma should always be at least receive a textual justification inside an annotation. A good practise is to submit such lemma to the FoCaL test tool to increase confidence.

\subsection{Around the Language}

All along the development cycle of a FoCaL program, the compiler keeps track of dependencies between species, their methods, the proofs, ... to ensure any modification of component will be detected and its impact will be reported on those depending of it.

FoCaL considers two types of dependencies:

- The decl-dependency: a method $A$ decl-depends on a method $B$ if the declaration of $B$ is required to express $A$.

- The def-dependency: a method (and more especially a theorem) $A$ def-depends on a method $B$ if the definition of $B$ is required to state $A$ (and more especially, to prove the property stated by the theorem $A$ ).

The redefinition of a function may invalidate the proofs that use properties of the body of the redefined function. All the proofs which truly depend on the definition are 
then erased by the compiler and must be done again in the context updated with the new definition. Hence an important point is the choice of the most interesting level in the hierarchy where to write a proof.

FoCaL currently supports two target languages: OCaml[10] and Coq[11]. Code generation towards OCaml allows to build an executable: all the logical aspects are discarded since they can't be expressed in this language and don't lead to executable code. Code generation towards Coq provides a formal model of the program, including computational and logical aspects: all computational methods and logical methods with their proofs are compiled. Thus the consistence of the whole FoCaL development can be checked by Coq.

However the compilation model (i.e. the structure of a collection, of a species, of a method) remains very simple. It is the same in both target languages and uses a few set of basic constructs: records (i.e. structures), functions and simple modules (not even functors).

Note that references are not currently allowed as it is not so easy to handle memory management at the proof level. However functional views of memory are used in several developments. We currently consider to add data-flow programming features and their logical counterparts to ease reactive systems development.

The tool called FOCDOC [3] automatically generates documentation, ensuring that the documentation of a component is always consistent with respect to its implementation. This tool uses its own XML format that contains information coming not only from structured comments (that are parsed and kept in the program's abstract syntax tree) and FoCaL concrete syntax but also from type inference and dependence analysis. From this XML representation and thanks to some XSLT style-sheets, it is possible to generate HTML or $\mathrm{HT}_{\mathrm{E}} \mathrm{X}$ files. In the same way, it is possible to produce UML models [5] as means to provide a graphical documentation (class diagrams) for FoCaL specifications. The use of graphical notations appears quite useful when interacting with end-users, as these tend to be more intuitive and easier to grasp than their formal (or textual) counterparts. This transformation is based on a formal scheme and captures every aspect of the FoCaL language, so that it has been possible to prove the soundness of this transformation (semantic preservation).

Although this documentation is not the complete safety case, it can helpfully contribute to its elaboration.

As a conclusion of this presentation, FoCaL is not at all a "All-in-One" language, it helps to define strict boundaries between phases of a development. Consistency between phases is ensured by a powerful dependency calculus and a proof system. FoCaL user is guided to maintain the global consistency of a complete development, even during the maintenance phase.

There exist several systems and languages having the same purposes like $\mathrm{B}[16]$, CASL[13], RAISE[15]... True comparison with these systems is out of the scope of this paper (but is considered in a forthcoming paper). It seems to us that the originality of FoCaL is its unified language along the different phases which allows to provide the assessor a complete package built in conformity with the related standards.

Differently from CASL[13] that is strongly oriented towards ADT, hence without effective representation of data and computation, FoCaL lets the user go until speci- 
fying them (and adds an abstraction layer to prevent users from having access to internal effective implementation). FoCaL produces executable code and related proofs whereas CASL emphasises on the specification phase despite the fact that institutions are provided to link specifications to some SML or Java code[14]. RAISE[15] provides a large range of specification and programming concepts. In contrast, FoCaL offers a limited set of concepts but they can be altogether translated to Coq to ensure a global consistency of the whole development.

\subsection{FoCaL development cycle}

As recalled in the introduction, the development of a critical system must follow a strict development cycle, compliant with standards. In [7] we proposed a development life cycle taking advantages of all features of FoCaL and compliant with the main Standards in the field of critical software development ([1], [2],...). It is based on a V-cycle, decomposed into five (mandatory) phases: requirements/specification, architecture/design, implementation and low level testing, integration/validation testing and the longest one, the maintenance phase. It also covers some transverse processes like generation of the documentation or formal traceability between phases. Transverse processes are processes that should be applied once during all phases. The main characteristics of this life cycle are :

- a strong boundary between phases, especially between the specification phase and the architecture/design one. Specification phase ends when all safety requirements can be proved using the functional requirements and the glue assumptions (see below). Architecture/design phase ends with the implementation of the functional requirements into executable code.

- the implementation phase consists in assembling collections and proving the glue assumptions made on the parametrised species.

- the use of the FoCaL dependencies calculus and documentation generation to generate formal traceability and to help maintenance.

In this paper, we mainly focus on requirements specification, architecture/design and implementation phases as we build the voter. Integration/validation testing phase is considered in the work of M. Carlier [6].

The development cycle is strongly based on the establishment of the specification through requirements expression. We distinguish four kinds of requirements:

- Functional requirements describe the relations between inputs and outputs of the system and what is expected on the behaviour of the system without referring to any specific solution.

- Non-functional requirements describe all constraints that the system must meet, like time and space bounds, safety integrity levels to achieve, portability needs... These requirements are pretty difficult to express by a first-order formula. They are put inside annotations, so are kept along the compilation process and figure in the compiled documentation.

- Safety requirements coming from the results of the safety studies. They ensure that the functional requirements will never trigger a Feared Event. They are considered as requirements on functional requirements. 
- As a species can be parametrised, proofs of functional and/or safety requirements sometimes need assumptions on the functions and properties of the collection parameters. These assumptions are called glue assumptions. They are proved at the coding level just before final building of the whole system.

All the requirements but some non-functional ones are expressed as FoCaL properties.

\section{Overview of the voter}

\subsection{Generic definition}

Sensors may exhibit various kinds of errors like bias offset, scale factor or transient faults due to sensitivity to spurious or environmental factors (temperature, pressure,...). Redundancy is one of the major techniques used to guard safety critical systems against such transient faults. There exists many kinds of redundancy, depending on which characteristics (safety, reliability or both) should be privileged for the system. Roughly speaking, each redundant component performs the same work and, when one of them fails, the voter has to detect it and to select an output value among the other, then has to go on providing the service.

Usually, a voter is used to elaborate the output from the input values given by the redundant components. Voters are used, for example, for temperature acquisition by multiple sensors in a boiler, or elaboration of the emergency brake signal of a train from several computer replicas... The basic principle of a voter is to compare its input values according to a given consistency relation, and then to output one value depending on a voting policy. The point is that, in redundant systems, the voter is the component that must be perfect (as far as possible obviously). A failure of the voter is considered as a major weakness of the system.

A voter system must fulfil three main requirements:

- reliable and correct choice of one non faulty input among its $n$ inputs ${ }^{5}$

- detection of errors on inputs

- localisation of the source of the error and report of a diagnosis related to it ${ }^{6}$

The elaboration of the output value follows a two-stages process:

1. the inputs comparison, which takes 2 or more inputs and compares them according to a "consistency law". There are many kinds of such consistency laws: strict equality, equality within a certain tolerance, most recent input, max or min values $\ldots$

2. the arbitration, by a voting policy algorithm which produces the output value. This algorithm is the heart of a voter and determines its classification and its main properties (majority vote, selection of the most restrictive vote, selection of the most recent value ...).

\footnotetext{
${ }^{5}$ We wilfully limit the voter specification to a function that returns one of its inputs. Other more complex voters can be found in [8].

${ }^{6}$ The third requirement is sometimes optional depending on which dependability characteristic is emphasised.
} 
A pre-processing of the inputs can also be performed by a filtering process. It analyses input values by in-line "acceptance tests" and eliminates values recognised wrong, a way to eliminate some transient faults. The inputs that succeed the filtering process are then sent to the voter ${ }^{7}$.

In the following, we focus on voters with 3 inputs. Our choice is motivated by the fact that 3 inputs is the minimal number for a voter to deal both with safety and reliability characteristics. Taking the case where agreement between 2 sensors is sufficient to ensure the required safety level of a system. Using a voter with 2 inputs meets the safety requirements. But, in case of one failure on a sensor, you need to stop or run your application in a degraded mode. Adding a third sensor permits to continue the service in case of one fault. Most of the time, the cost of the third sensor is far less than the unavailability of the whole system. This kind of voter is a widespread voter architecture in safety critical systems (i.e. Triple Modular Redundancy).

Our choice could be even more generic. It is indeed possible to specify an " $n$ out of $m$ " voter and then to instantiate $n$ and $m$ with the needed value. But this solution needs to highly complicate the voter specification and implementation for a small added value. Indeed only some $n$ and $m$ values are generally used; 1002 for reliability, 2oo2 for safety, 2003 or 2004 for safety and reliability and the concerns are so different that this "sharing by genericity" is just useless.

\subsection{The 2003 voter specification}

The 2003 voter, used for our example, selects one value from three independent inputs if at least two of them are consistent. Moreover, we also want to detect the faulty value. So, a second output is added to the voter in order to qualify the first result. Table 1 summarises all cases, described as follows:

- perfect_match: the three inputs are consistent, the value and index of one of them are returned.

- partial_match: two of the three inputs are consistent together, but the third one is not. One of the consistent values and the index of the inconsistent one are returned. This enables identifying a failure on this input.

- range_match: One input is consistent with the two others which are mutually inconsistent. The consistent value and the associated index are returned. This can arise when the consistency law is not transitive (i.e. equality within a tolerance). In this case, the system can go on working with the most plausible value.

- no_match: all the inputs are inconsistent two per two. The voter cannot take a decision since the majority rule is not applicable.

The specification of the no_match case seems, at first sight, satisfactory: no value is output as there is no good candidate. At the specification level, this behaviour is acceptable, but a choice has to be made during the design phase: the component connected to the voter is waiting for two values (the index of the component and the flag). It will be its own concern to decide what to do with the first output, according to the second.

${ }^{7}$ Filtering is different from the input comparison as it works on one single input in opposition to the consistency law which compare at least two inputs. 


\begin{tabular}{|c|c|c||c|c|l|}
\hline \multicolumn{2}{|c|}{ Consistency between inputs } & Returned & \multicolumn{2}{|c|}{ Diag } \\
\cline { 1 - 1 } v1 and v2 & v1 and v3 & v2 and v3 & Value & Index & Qualifier \\
\hline \hline Yes & Yes & Yes & v1 & sensor_1 & perfect_match \\
\hline Yes & Yes & No & v1 & sensor_1 & partial_match \\
\hline Yes & No & Yes & v2 & sensor_2 & partial_match \\
\hline No & Yes & Yes & v2 & sensor_3 & partial_match \\
\hline Yes & No & No & v1 & sensor_3 & range_match \\
\hline No & Yes & No & v3 & sensor_2 & range_match \\
\hline No & No & Yes & v2 & sensor_1 & range_match \\
\hline No & No & No & $?$ & $?$ & no_match \\
\hline
\end{tabular}

Table 1. Transfer function

\subsection{The 2003 properties}

Functional requirements should describe the voting policy. Each line of the table 1 is transposed in a functional requirement. For example, for a perfect match, the requirement is:

$$
\begin{aligned}
& \forall v 1, v 2, v 3 \text { in value, consistency }(v 1, v 2) \wedge \\
& \text { consistency }(v 1, v 3) \wedge \text { consistency }(v 2, v 3) \\
& \Rightarrow \operatorname{voter}(v 1, v 2, v 3)=(v 1,1, \text { perfect_match })
\end{aligned}
$$

Table 1 makes also assumptions on symmetry and reflexivity of the consistency ${ }^{l a w}{ }^{8}$. This leads to define the following glue assumptions:

$$
\begin{aligned}
& \forall v 1, v 2 \text { in value, consistency }(v 1, v 2) \Rightarrow \text { consistency }(v 2, v 1) \\
& \text { and } \\
& \forall v \text { in value, consistency }(v, v)
\end{aligned}
$$

A voter should also meet some safety requirements. Whatever is the order of the input values, the voter has to return a compatible output value. Thus a notion of "compatible output value" is introduced by properties P1 and P2. P1 says that if input values can be compared and output values are consistent and qualifiers are the same then the output values are compatible. P2 says that by default all output values are compatible for inconsistent input values (i.e. there is no choice made for inconsistent input values).

$$
\begin{aligned}
& \mathrm{P} 1: \forall v_{a l}, \text { val }_{b} \text { in value, } \forall \text { qual in qualifier, } \\
& \text { qual } \neq \text { no_match } \wedge \text { consistency }\left(\text { val }_{a}, \text { val }_{b}\right) \\
& \Rightarrow \text { compatible }\left(\text { val }_{a}, \text { val }_{b}, q u a l, q u a l\right) \\
& \text { P2 : } \forall v_{a l}, \text { val }_{b} \text { in value, } \\
& \text { compatible }\left(\text { val }_{a}, v v_{b}, \text { no_match, no_match }\right) \\
& \text { and } \\
& \forall v 1, v 2, v 3, v_{a}, v_{a} l_{b} \text { in value, } \text { qual }_{a}, \text { qual }_{b} \text { in qualifier } \\
& \operatorname{voter}(v 1, v 2, v 3)=\left(v_{a l_{a}}, \ldots, q u a l_{a}\right) \Rightarrow \\
& \operatorname{voter}(v 2, v 1, v 3)=\left(v_{a l_{b},}, q_{q u a l_{b}}\right) \Rightarrow \\
& \text { compatible }\left(\text { val }_{a}, \text { val }_{b}, \text { qual }_{a}, \text { qual }_{b}\right)
\end{aligned}
$$

\footnotetext{
${ }^{8}$ Note that the voter does not need transitivity for the consistency law. Use of a transitive consistency law will remove the partial_match qualifier.
} 
Another safety requirement allocated to a voter is that the output value is always one of the inputs.

$\forall v 1, v 2, v 3$ in value,

$$
\operatorname{vote}(v 1, v 2, v 3)=v 1 \vee \operatorname{vote}(v 1, v 2, v 3)=v 2 \vee \operatorname{vote}(v 1, v 2, v 3)=v 3
$$

\section{Development of the voter}

\subsection{Global architecture}

The voter specification closely follows the description given in Section 3. Indeed separation of the voting policy and the inputs management eases reuse and independent evolution of parts of the voter. The whole specification of the voter is thus split into two major parts. The part concerning the voting policy is introduced by the species Voter (see fig. 1) which specifies the voting policy algorithm and the glue assumptions made on the inputs of the voter, represented by parameters. The second part, which concerns values, is itself split into two parts, the first one describing what is supposed/needed on the "basic" types (like naturals, integers, booleans) and functions on them, the second one (the species Values) specifying inputs as "complex values" (like integers with tolerance, integers modulo $n$ ) built upon basic types and ensuring that glue assumptions could be satisfied. Consistency between the two parts is guaranteed by the FoCaL dependency and proof mechanisms.

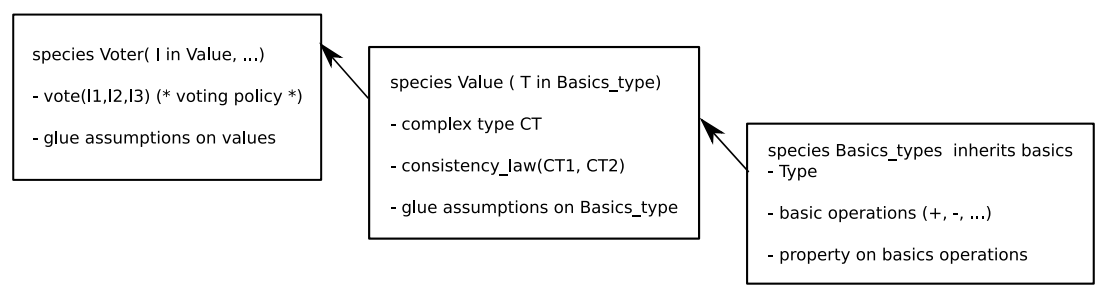

Fig.1. Voter decomposition

\subsection{The voting policy}

Specification phase The aim is to specify the data-flow interfaces and the requirements for the system without referring to any specific solution. In FoCaL, data-flow interfaces are encoded by functions declarations and requirements by properties on these functions.

Specification of the voting policy is performed with a two levels approach. The first level corresponds to the specification of a most general voter. It gives the signatures and the generic properties of all 2003 voters, making no assumption on the voting policy. 
The second level, derived from the first one, specifies the voting policy and gives the proof of the generic properties.

The specification of the generic 2003 voter is represented by a species "Voter_2oo3" with two parameters: a collection $\mathrm{V}$ for the values submitted to the vote, a collection $\mathrm{Di}$ ag for diagnosis prescriptions. The species provides a single signature vote. It also gives the safety requirements allocated to the voter. In order to ease reading, we add a shortcut to extract the value from of output value.

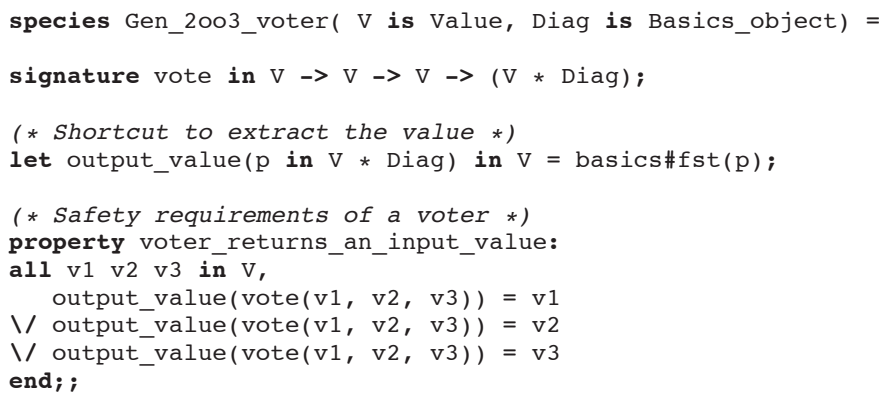

A species can be derived from this generic species for any choice of a voting policy. These derived species define the "voting policy", the glue properties required on the input values (through the collection parameter of interface Value) and give a proof of the generic properties. As an example, see the specification of a majority voter with identification of the faulty inputs as defined in section 3 .

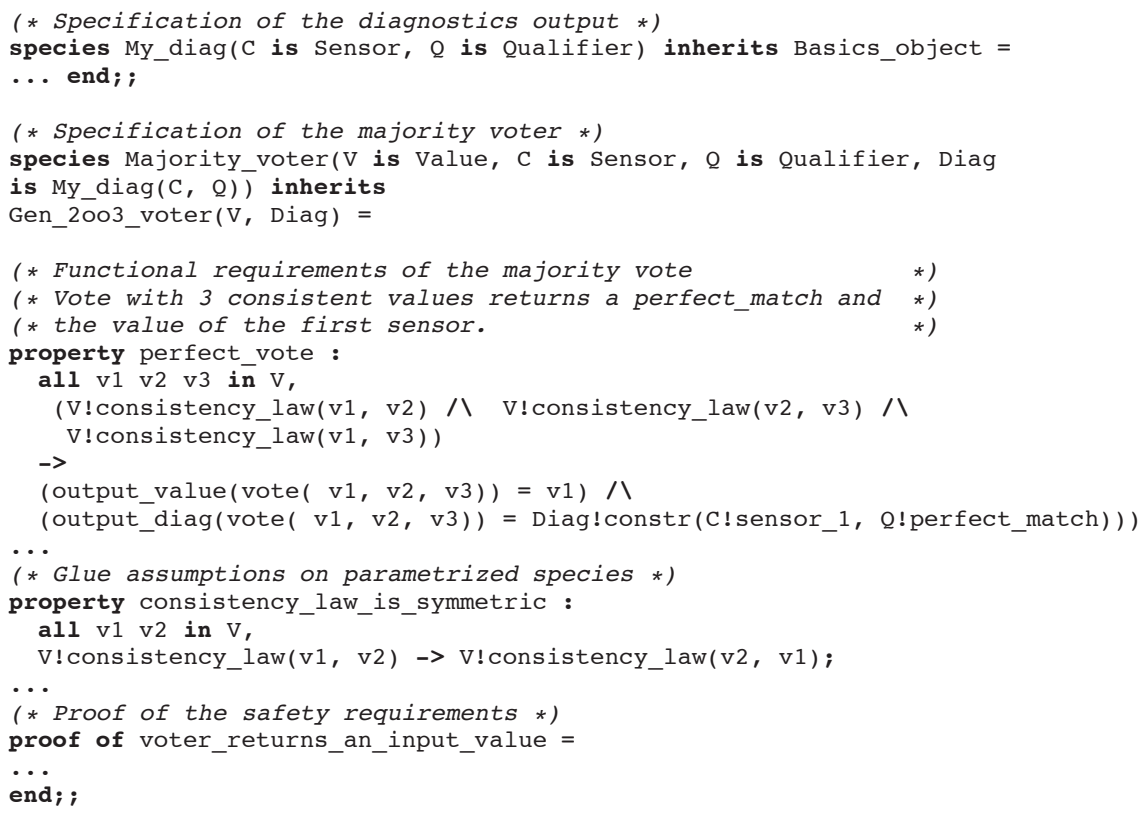

To create the specification of the "majority voter", we use two main features of the FoCaL language. First, we use the FoCaL inheritance mechanism to create a species 
having all declarations, definitions and properties defined in the species Gen_2003_ voter. Then, we instantiate parameters of Gen_20o3_voter with a more specific species (i.e. My_diag). Note that, the dependency calculus ensures consistence between the different occurrences of formal and effective parameters.

The new species contains the functional specification of the 2003 majority voter, the glue assumptions on parameters and the proof of safety properties under those assumptions (done with Zenon). We can compile the FoCaL model to an OCaml file and a Coq file. The first one contains only typed functions declarations that can be used as an external interface for a development. The second one contains a proof term of the safety properties that can already be checked by the Coq prover.

Architecture/design phase This phase introduces the architectural choices to answer the specification requirements. It provides definition of the functions and the representation of the species. From the majority voter specification, we have sufficient information to propose an implementation of the functional requirements and to provide a proof that this code fulfils them.

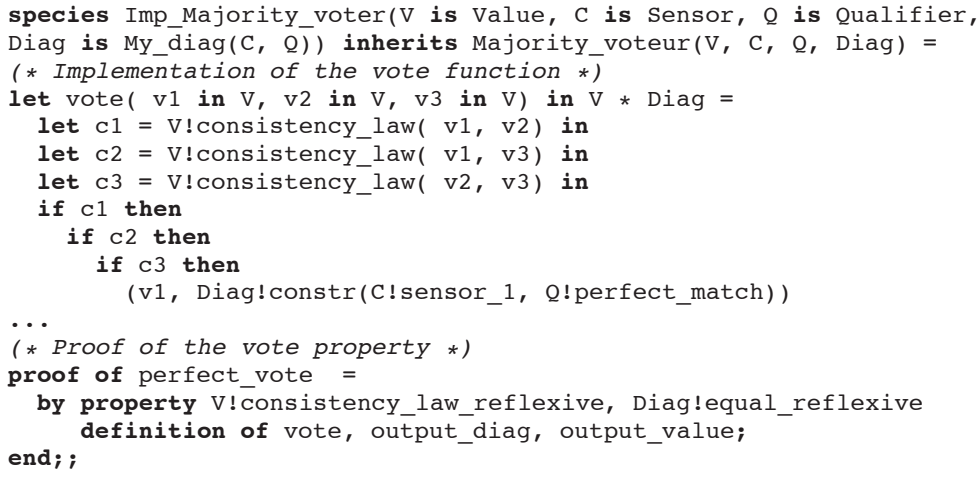

Transition between specification and design is also made using inheritance. Here parameters are kept while a definition is provided to the declaration, using the FoCaL functional language. Proofs of the functional properties are made using Zenon. Giving a FoCaL interface (and thus at least a specification species) for the input values, the sensor and the qualifier, this species can be compiled: type-checking can be done and a translation of the whole species contents (including Zenon proofs) into a Coq term can be obtained to be immediately assessed. Thus we have a species that implements a 2003 majority voter, which can be used with any kind of input values respecting the required glue properties.

Multiple inheritance can be used at design level to provide an existing representation to a specification species. For instance the implementation species Imp_vote_status is a "merge" of two species: the specification species Sp_vote_status which provides the functional requirements and the species Integers which provides the representation and its basic properties. The dependency calculus ensures compatibility between these inherited species.

(* Specification species for a set of vote status *)

species Sp_vote_status inherits Setoid = 


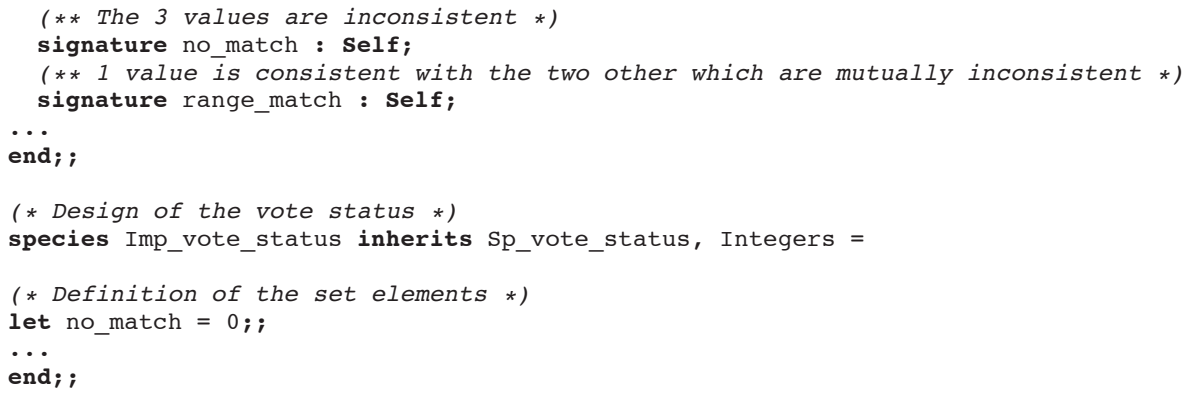

Definitions can also be changed at any level to fit new needs. In this case, the FoCaL compiler computes all definitions and properties impacted by the redefinitions and asks to provide a new proof of the impacted properties.

\subsection{Building a voter}

At this stage, we have several species representing all components of the voter. In order to obtain runnable code a species should be transformed into a collection. Thus a voter collection has to be created from the voter species and collections representing the values and the diagnosis. Firstly, we choose the nature of input values (here the collection Coll_int_with_tol), finalise the diagnosis (here the collection Coll_my_diag). Secondly, we create a species which inherits from the implementation of the voter applied to effective parameters and we provide a proof for the glue assumptions. This step ensures "compatibility" between species. Then, as the new species is now complete, we can create a collection.

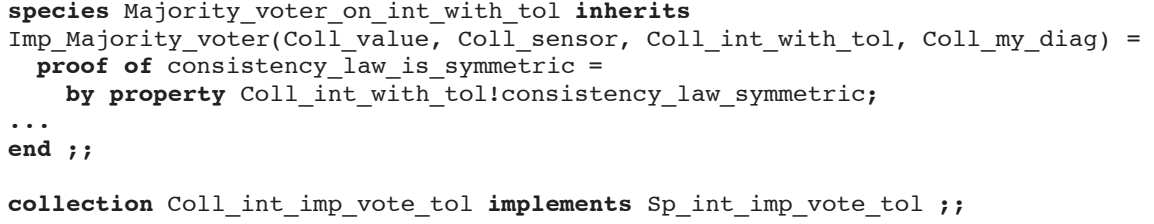

The collection Coll_int_imp_vote_tol is ready to use.

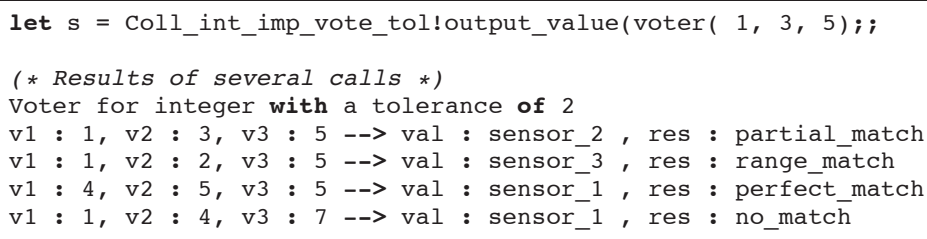

When a component is a COTS (Commercial Off The Shelf) or a very low level component as I/O drivers, one cannot produce a proof of its functional properties. In the same way, we do not want to prove well known or highly used components. In order to consolidate our confidence level of such components, works are currently performed by M. Carlier[6] to generate test cases from FoCaL functional requirements. These test cases can be ran on the external component to validate it. Verification of the voter using 
M. Carlier tool has been achieved. It shows that the FoCaL life cycle we propose is fully compliant with the validation of external components.

\subsection{Re-usability}

Many other implementations can fit the generic voter. For example, S. Dajani-Brown proposes a 2003 voter for avionics purpose[9]. This voter is based on a classical 2003 architecture with an high availability (voter provides an output even when only one input is available). Following our development cycle, we implemented this voter within FoCaL by changing the voting policy and the value representation. Then we carried out successfully all the proof of the generic voter properties (voter always returns one of the input values, voter is insensitive to inputs order).

\section{Conclusion and further works}

This paper illustrates how a safety life cycle can be developed using FoCaL features like inheritance, parametrisation by collections, properties and proofs. This development process respects boundaries between development phases, produces a certified and efficient code, is compliant with standards and thus, eases the assessment process required before commissioning. Several other examples have been developed following the same methodology like hierarchical automata's, physical input acquisition... These developments give similar results on the development process.

In this paper, due to a lack of space, we only gave a short glimpse on FoCaL testing. However we used the testing tool (still in development), not only to do classical tests on outputs of the voter but also when encountering some difficulties in proofs, to validate expression of some requirements. We appreciated a lot to have at our disposal, at any stage of the development cycle, a proof tool and a test tool working on the same expressions. This is indeed a FoCaL feature which is of great help when expressing some requirements, when wondering about the validity of some lemmas, when some proofs are out of reach. In this last case, some of the corresponding statements are considered as axioms during proof process, a point which weaknesses the logical approach but is unavoidable in practice, thus increasing confidence by testing the statement is welcomed.

More generally, we emphasise the great facilities given by the coordination of FoCaL programming features (typing, inheritance, late binding, etc.) and FoCaL logical features (statements, proofs) through syntactical means controlled by dependency calculus. Indeed, having the possibility to introduce a statement using names not yet associated to a definition and to prove it under hypothesis submitted to a delayed proof obligation, allows to detect anomalies sooner in the life cycle and the diagnostic provided by the compiler helps to repair them. Yet it is possible to criticise the choice of a functional language for source code. Having no notion of internal state for species can be considered as a weakness. But first, there is no difficulty to translate, if needed, FoCaL definitions into some imperative language: the compiler uses only very basic features of programming languages (simple records and modules). Second, a lot of static analysers rebuild a functional version of imperative code to perform their analyses. In FoCaL this functional version is directly at hand, a point which will be exploited to integrate static 
analysers in FoCaL, in the near future as the new compiler was conceived to easily include such extensions.

Using the theorem prover Coq as an assessor while having near all proofs quite automatically done with Zenon and having the choice of either doing the remaining ones directly in the Coq environment build by the FoCaL compiler or assuming their statement appears as a good compromise between the confidence level and the cost of the development. Yet the choice of Coq can be questioned. As the compiler does not use truly specific features of Coq, other theorem provers based on type theories and a flavor of Curry-Howard isomorphism can be chosen.

\section{References}

1. "Railway Applications - Communications, Signalling and Processing Systems - Software for Railway Control and Protection Systems", Standard Cenelec EN 50128, 1999

2. "Functional safety of electrical/electronic/programmable electronic safety-related systems", Standard IEC-61508, International Electrotechnical Commission, 1998

3. Manuel Maarek and Virgile Prevosto, "FoCDoC: the documentation system of FoC", in Proceedings of Calculemus, September 2003

4. Richard Bonichon, David Delahaye and Damien Doligez, Zenon : An Extensible Automated Theorem Prover Producing Checkable Proofs", LPAR, pages 151-165, 2007

5. D. Delahaye, J.F. Etienne and V. Donzeau-Gouge. "Producing UML Models from Focal Specifications: An Application to Airport Security Regulations" in 2nd IFIP/IEEE International Symposium on Theoretical Aspects of Software Engineering pp. 121-124, 2008

6. M. Carlier and C. Dubois, "Functional Testing in the Focal environment", in Test And Proof (TAP'2008), B. Beckert and R. Hahnle eds, LNCS 4966, pages 84-98, April 2008

7. P. Ayrault, T. Hardin, F. Pessaux, "Development life cycle of critical software under FoCaL" in TTSS'08, harnessing Theories for Tools Support in Software, Istanbul 2008

8. Paul R. Lorczack, et al, "Theoretical Investigation of Generalized Voters for Redundant Systems", Digest of Papers FTCS-19: The Nineteenth International Symposium on Fault-Tolerant Computing, 1989, pp. $444-451$.

9. Samar Dajani-Brown, Darren Cofer, Amar Bouali, "Formal verification of an avionics sensor voter using SCADE", Formal Techniques, Modelling and Analysis of Timed and FaultTolerant Systems, 2004, pp. 5 - 20.

10. Xavier Leroy with Damien Doligez, Jacques Garrigue, Didier Rémy and Jérôme Vouillon. "The Objective Caml system, documentation and user manual. release 3.11.", Documents include with the Objective Caml distribution, INRIA 11/2008

11. Yves Bertot, Pierre Castéran "Coq'Art: The Calculus of Inductive Constructions", Series: Texts in Theoretical Computer Science. An EATCS Series

12. Catherine Dubois, Thérèse Hardin and Véronique Viguié Donzeau-Gouge, "Building a certified component within FoCaL", Trends in Functional Programming Vol. 5, Intellect, 2006, pp. $33-48$.

13. E. Astesiano, M. Bidoit, H. Kirchner, B. Krieg-Brückner, P.D. Moss, D. Sannella, A. Tarlecki, "CASL: the Common Algebraic Specification Language", Theorical Computer Science 286, 2002, pp 153 - 196

14. D. Aspinall and D. Sannella, "From specification to code in CASL" AMAST, 2002

15. The RAISE Method Group, "The RAISE Development Method", Prentice Hall International, 1995

16. J.R. Abrial, "The B-Book - Assigning Programs to meanings", Cambridge University press, 1996. 